\title{
Explanation of atomic displacement around lattice vacancies in diamond based on electron delocalization
}

\author{
M. Heidari Saani ${ }^{1,2, a}$, H. Hashemi ${ }^{3,4}$, A. Ranjbar ${ }^{5}$, M.A. Vesaghi ${ }^{5}$, and A. Shafiekhani ${ }^{6}$ \\ 1 Semiconductor Component Industry, P.O. Box 19575-199, Tehran, Iran \\ 2 Physics Group, Maleke Ashtar University, P.O. Box 83145-115, Shahin Shahr, Iran \\ 3 Fachbereich Physik, Martin-Luther-Universität, Halle-Wittenberg, Friedemann-Bach-Platz 6, D-06099 Halle, Germany \\ 4 International Max Planck Research School for Science and Technology of Nanostructures, Weinberg 2, D-06120 Halle, \\ Germany \\ 5 Department of Physics, Sharif University of Technology, P.O. Box 11365-9161, Tehran, Iran \\ ${ }^{6}$ Department of Physics, Alzahra University, Vanak, Tehran, 1993891167, Iran
}

Received 19 June 2007 / Received in final form 10 March 2008

Published online 12 September 2008 - (C) EDP Sciences, Società Italiana di Fisica, Springer-Verlag 2008

\begin{abstract}
The relationship between unpaired electron delocalization and nearest-neighbor atomic relaxations in the vacancies of diamond has been determined in order to understand the microscopic reason behind the neighboring atomic relaxation. The Density Functional Theory (DFT) cluster method is applied to calculate the single-electron wavefunction of the vacancy in different charge states. Depending on the charge and spin state of the vacancies, at outward relaxations, 84-90\% of the unpaired electron densities are localized on the first neighboring atoms. The calculated spin localizations on the first neighboring atoms in the ground state of the negatively charged vacancy and in the spin quintet excited state of the neutral vacancy are in good agreement with Electron Paramagnetic Resonance (EPR) measurements. The calculated spin localization of the positively charged vacancy contrasts with the tentative assignment of the NIRIM-3 EPR signal to this center in (p-type) semiconductor diamond. The sign of the lattice relaxation in the diamond vacancy is explained based on the effect of electron delocalization on nearest-neighbor ion-ion screening, and also its effect on the bond length of neighboring atoms.
\end{abstract}

PACS. $61.72 . \mathrm{Bb}$ Theories and models of crystal defects - 61.72.Ji Point defects (vacancies, interstitials, color centers, etc.) and defect clusters - 71.55.-i Impurity and defect levels

\section{Introduction}

Experimental measurements and theoretical calculations $[1,2]$ suggest that after creation of a vacant site in a semiconductor lattice, the electrons of broken bonds and also surrounding atoms change their configurations to attain a new configuration with a new minimum energy. The system must reconfigure itself to reach a new equilibrium in which the neighboring atoms are relaxed and the unpaired electrons delocalized. Since a mono-vacancy in a diamond lattice is the most fundamental defect, a number of theoretical calculations have already been reported on their structure [3-7]. Recently, a qualitative explanation for the deviation of unpaired electronic density from directional $s p^{3}$ distribution, and also delocalization of vacancy electrons was reported based on the ab-initio DFT method [8]. Furthermore, by including the effect of lattice relaxation into the many-particle Hamiltonian of the

\footnotetext{
a e-mail: heidaris@yahoo.com
}

system [9], the experimental evidences concerning electron paramagnetic resonance (EPR) measurements on the neutral vacancy in diamond were explained [10].

In order to understand the underlying microscopic mechanism of the lattice relaxation, we used an ab-initio Density Functional Theory (DFT) cluster method to calculate the variation of vacancy electron localization on the first, second and third neighboring atoms of the vacancy with respect to the lattice relaxation. The ground states of the neutral $\left(V^{0}\right)$, negative $\left(V^{-}\right)$, and positive $\left(V^{+}\right)$charge states of the vacancy in diamond in addition to the spin quintet $(S=2)$ excited state of $V^{0}$, have been investigated. The percentages of the unpaired electron (spin) localization on the first neighboring atoms were calculated and the results were compared with available EPR experimental data. We discuss the sign of the relaxation of the first neighboring atoms and also the negligible relaxation of the second neighboring atoms based on the delocalization of the vacancy electrons. 


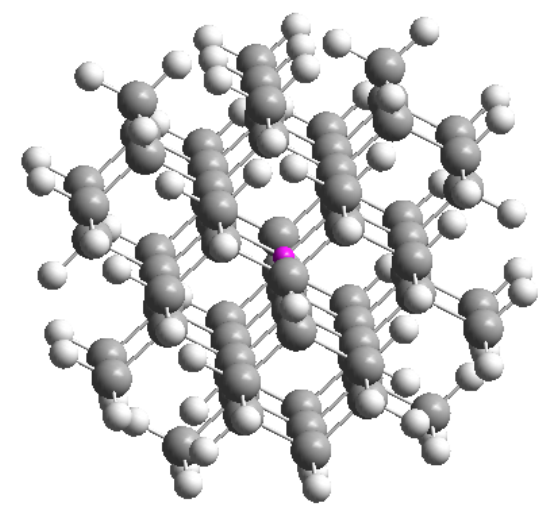

Fig. 1. The $\mathrm{C}_{70} \mathrm{H}_{84}$ hydrogen terminated cluster with a vacancy at its center that was used to simulate the diamond vacancy.

\section{Calculation method}

As already noted above, the calculations reported here were performed within the framework of the density functional theory (DFT) cluster method to calculate the wavefunctions of unpaired electrons in different charge states of the vacancy. The lattice vacancies in diamond were simulated with a missing atom at the center of a $\mathrm{C}_{70} \mathrm{H}_{84}$ hydrogen terminated cluster $[4,5]$. The cluster with the vacancy at its center is shown in Figure 1.

We used the Gaussian 98 code for the self-consistent calculations of DFT [11]. The code gives eigenfunctions of Kohn-Sham energy levels in a real-space representation for crystalline point defects $[12,13]$. In this representation, energy eigenfunctions are expanded using a basis set of atom-centered Gaussian functions and thus is capable of high numerical accuracy for an all-electron calculation. In this calculation, we used an atom-centered $6-31++\mathrm{G}$ extended basis set for the first and second neighboring shells of atoms around the vacancy, a $3-21 \mathrm{G}$ basis set for the third neighboring atoms and a minimal STO-3G basis set for the remaining atoms [14]. In parallel, we also performed a calculation using STO-3G minimal basis set for all atoms of the cluster and found qualitatively similar results for all charge states of the cluster. The perfect cluster was relaxed and the equilibrium lengths of $\mathrm{C}-\mathrm{C}$ and surface $\mathrm{C}-\mathrm{H}$ bonds were found to be $1.535 \AA$ and $1.05 \AA$, respectively. Self-consistency of calculations was achieved when the total energies converged to within $10^{-5}$ a.u.. Exchange and correlation effects were described by the B3LYP functional. This functional includes the Becke three parameter hybrid exchange functional [15].

In different charge states of the defect, the eigenfunction of the highest filled energy level was cut off at the fourth neighboring atoms, and the weak tail of the wavefunction on the fourth neighboring and farther atoms is neglected. The calculated charge (spin) density on the fourth neighboring atoms is very small (less than 1\%) However, from the fifth neighboring atoms the probability of finding electronic charge density starts to increase. The increment reaches its maximum value just at the surface carbons. This residual charge density originates from the hydrogen surface saturators. Although surface saturators eliminate undesirable band-gap states, the difference between the electron-affinity of carbon and hydrogen induces undesirable negative charge into the volume of the cluster. The cutting of the wavefunction at the fourth neighboring atom separates it from the wavefunction tail of the electrons of the hydrogen-terminated surface. EPR measurements indicate that at the third neighboring atoms the spin density is too small, where the HFI interaction of the unpaired electron (spin) density becomes comparable to the direct spin-spin interaction of the ${ }^{13} \mathrm{C}$ isotopes carbon nucleus. This effect makes the HFI data on the third and farther neighboring atoms unreliable [16]. We conclude that considering three neighbor shells of atoms in the calculation is reasonable and sufficient for explaining the EPR measurement data.

The localized charge (spin) density on the first, second and third neighboring atoms was calculated by volume integration over the square of the truncated wavefunction.

$$
\text { Localization }=\int_{r_{1}}^{r_{2}} \Psi(\vec{r}) \Psi^{*}(\vec{r}) d^{3} \vec{r} / \int_{0}^{r_{0}} \Psi(\vec{r}) \Psi^{*}(\vec{r}) d^{3} \vec{r} \text {. }
$$

In equation $(1), \Psi(\vec{r})$ is the truncated wavefunction of the unpaired vacancy electrons, $r_{1}$ and $r_{2}$ are the radial boundaries of the specific neighboring shell and $r_{0}$ is the cutting radius of the wavefunction.

\section{Results}

In Figures 2-5, we have shown results of the charge distribution over different neighboring shells around the vacancy in the ground state of the $V^{0}$, the spin quintet $\left(S=2\right.$ ) excited state of the $V^{0}$, the ground states of the $V^{-}$, and the ground state of the $V^{+}$, respectively. The magnitudes of the electron localization for different neighboring shells were calculated using equation (1).

As one can see from these figures, the trend of variation of charge on each neighboring shells of atoms around the vacancy is the same for all investigated charge states of the vacancy. In these figures, at $-20 \%$ inward relaxation of nearest-neighbor (NN) atoms, there is a comparable charge localization on the first and second neighboring atoms. At this relaxation, the amount of the charge localization on the third neighboring atoms is negligible. By outward displacement of NN atoms, there is a significant migration of charge from the second to the first and third neighboring atoms. In other words, by outward displacement of NN atoms from $-20 \%$ to $0 \%$ the amount of charge on the first neighboring atoms increases slightly, whereas the charge on the second neighboring atoms decreases rapidly. On the third neighboring shell of atoms we observe a slight increase in the localized charge by the outward relaxation. The rapid decrease in the calculated electron localization on the first neighboring atoms with inward relaxation can be attributed to the strong coulombic repulsion between vacancy electrons at shorter 


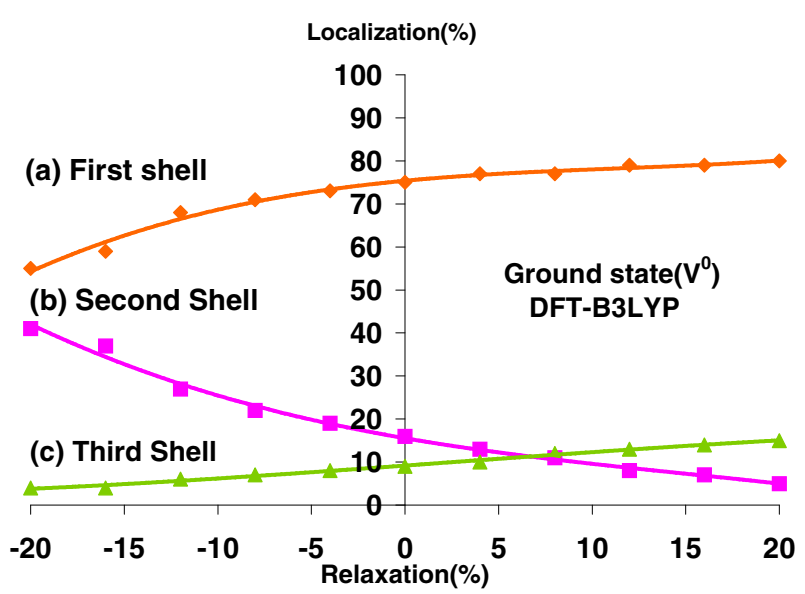

Fig. 2. The variation of electron localization on the first, second, and third neighboring atoms in the non-paramagnetic ground state of $V^{0}$ with outward (positive) and inward (negative) relaxation of $\mathrm{NN}$ atoms.

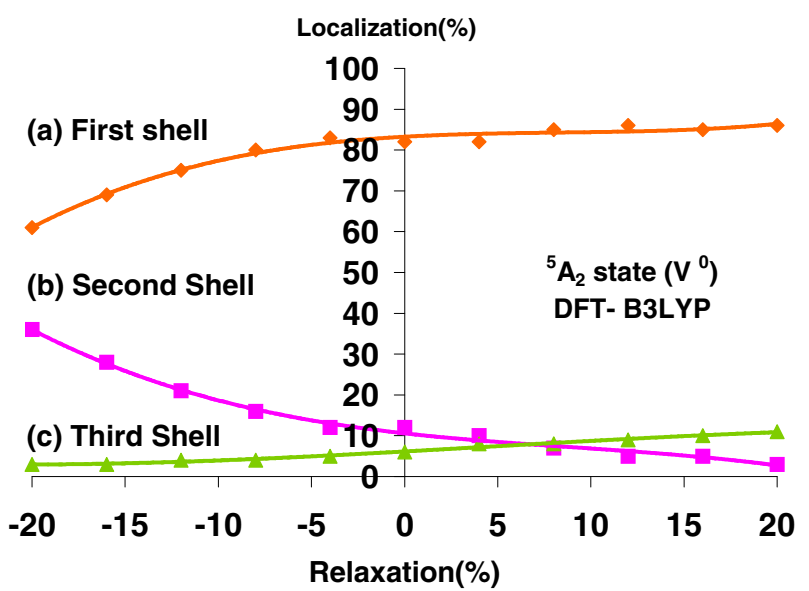

Fig. 3. The variation of electron localization on the first, second, and third neighboring atoms in the spin quintet $(S=2)$ excited state of $V^{0}$ with outward (positive) and inward (negative) relaxation of $\mathrm{NN}$ atoms.

distances. At the zero relaxations in Figures $2-5$, the amount of charge on the first neighboring atoms reaches its maximum value and remains almost constant at larger outward relaxations. In the zero relaxation, the amount of charge on the second and third neighboring atoms becomes comparable. By increasing the outward displacement of NN atoms from $0 \%$ to $20 \%$, migration of charge from the second to the third neighboring atoms reduces the charge localization on the second and increases the charge localization on the third neighboring atoms.

For all charge states of the diamond vacancy, at a specific size of the outward relaxation, between $4 \%$ and $8 \%$, the magnitude of charge on the third neighboring atoms exceeds that one on the second neighboring atoms. This indicates that in outward relaxations, the variation of charge in space is not monotonic. In outward relaxations, there is a crossing point where above it, the amount of the charge density on the third neighboring atoms is larger than that on the second neighboring atoms and, in relax-

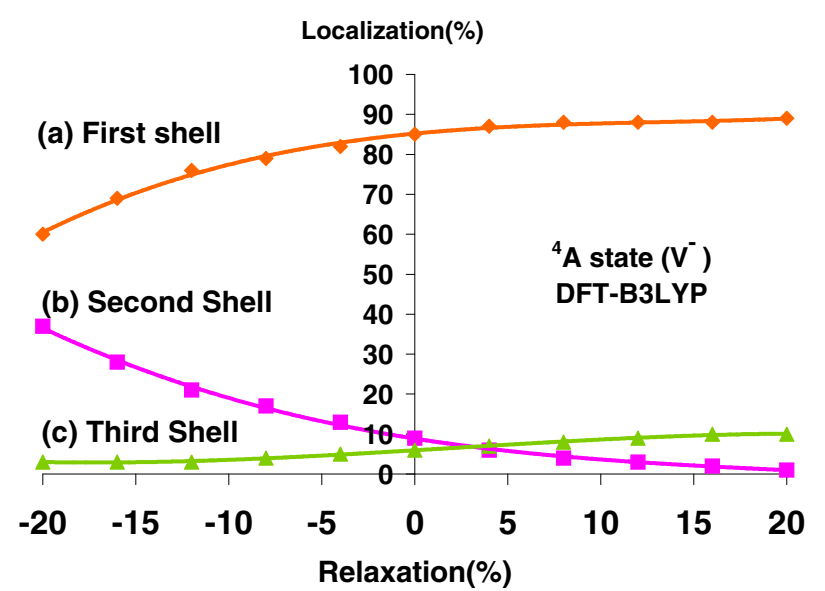

Fig. 4. The variation of electron localization on the first, second, and third neighboring atoms in the spin polarized $(S=$ $\frac{3}{2}$ ) ground state of $V^{-}$with outward (positive) and inward (negative) relaxation of $\mathrm{NN}$ atoms.

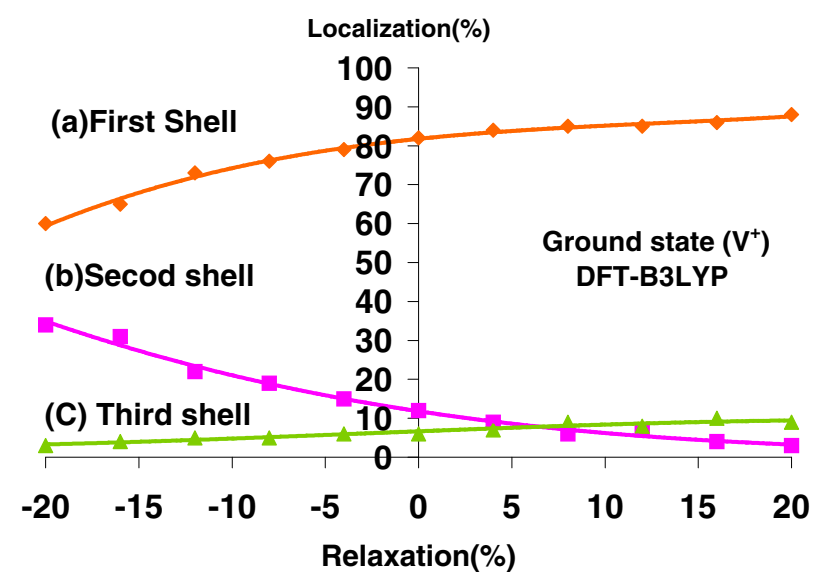

Fig. 5. The variation of electron localization on the first, second, and third neighboring atoms in the spin polarized $(S=$ $\frac{1}{2}$ ) ground state of $V^{+}$with outward (positive) and inward (negative) relaxation of $\mathrm{NN}$ atoms.

ations larger than the crossing point, the magnitude of the charge (spin) density on the first neighboring atoms does not vary significantly with relaxation.

\section{Discussion}

\subsection{Comparison with EPR measurements}

The magnitude of the electron localization around the lattice vacancy of a semiconductor decreases with increase of the semiconductor band-gap [16]. Therefore, we expect a small magnitude of vacancy electron delocalization in diamond. Experimental information about charge distribution and lattice relaxation around the lattice vacancy comes from EPR measurements. Our calculated spin localizations in the ground state of $V^{-}$and also in the spin quintet excited state of $V^{0}$ on different neighboring atoms 
are $89 \%$ and $85 \%$ respectively. These values are in very good agreement with 100\% [16] and 75\% [10] localizations the reported by EPR measurements on the ground and excited states of $V^{-}$and $V^{0}$, respectively. The lattice relaxation in the spin quintet excited state of $V^{0}$ $(15 \%)[10]$ is larger than that one in the ground state of $V^{-}(12 \%)[10,16]$. Our result agrees well with this experimental conclusion.

The hyperfine interaction (HFI) measurement on the second neighboring atoms of the vacancy is very difficult due to the weak tail of the unpaired electron wavefunction on these atoms [16]. Our results summarized in Figures 14 , predict the amount of unpaired electron density on the second neighboring atoms for different charge states of the vacancy to be between $4 \%$ and $7 \%$.

Now we discuss the probability of finding the positive charge state of the lattice vacancy $\left(V^{+}\right)$in diamond. This charge state of the vacancy is expected to be created, in ptype boron- doped, diamond under certain condition [17]. So far, however, no optical transition associated with a positive vacancy has been reported [17]. The EPR center NIRIM-3, produced by electron irradiation or proton implantation of synthetic boron- doped (type-IIb) diamond, has been tentatively attributed [18] to $V^{+}$with $S=\frac{1}{2}$. However, the unpaired spin density on the first neighboring atoms of NIRIM-3 is only about $17 \%$, showing either that the unpaired electron is highly delocalized, because its energy level is very close to the valence band, or that the interpretation is incorrect and the majority of the unpaired electron density resides on some central substitutional impurity with zero nuclear spin [2]. Our results did not show any significant difference in the delocalization of the unpaired electron density of $V^{+}$and two other charged states of the vacancy. We obtained about $88 \%$ spin localization on the first neighboring atoms of $V^{+}$, in the unrelaxed and symmetrically outward relaxed regimes. The results clearly contrast with the tentative assignment of the NIRIM-3 EPR center to $V^{+}$in semiconductor diamond.

\subsection{Effect of NN ion-ion screening on the relaxation of the vacancy}

The variation of vacancy electron localization calculated on different neighboring atoms is not symmetric with respect to the relaxation sign. The magnitude of the charge localization on the first neighboring atoms does not vary with outward relaxation. This stationary behavior of the vacancy electron localization on the first neighboring atoms results in a stationary character for the ion-ion screening of NN atoms under outward relaxation.

However, in the inward relaxations, we observe a continuous increase in the delocalization due to coulombic repulsion between vacancy electrons. This effect is accompanied by a decrease in the ion-ion screening and contrasts with the inward relaxation due to the increasing NN ionion repulsive potential. In conclusion, the inward lattice relaxation is not consistent with the results of electron localization variation with NN atom relaxation. The results are also in agreement with the reported crucial role of the ion-ion interaction in outward relaxation of the vacancy $[9]$.

\subsection{Effect of vacancy electrons delocalization on the surrounding $\mathrm{C}-\mathrm{C}$ bond lengths}

The increase in electron delocalization with inward relaxations is accompanied by an increase in charge density on back bonds, particularly in bonds between the first and second neighboring atoms, and $\mathrm{C}-\mathrm{C}$ bond lengths between two neighboring shells are reduced. The vacancy electron delocalization increases electronic charge density on the bonds between second and third neighboring atoms slightly, but it reduces the bond length between second and third neighboring atoms. In this way, in inward relaxations, similar to the NN ion-ion screening effect, the charge density on the back bonds increases. This contrasts with the inward relaxation and favors the outward lattice relaxation of NN atoms. Therefore, electron delocalization is consistent with the outward lattice relaxation and is not consistent with the reported inward lattice relaxation around the vacancy in diamond $[19,20]$.

The explanation of lattice relaxation based on electron delocalization can also explain the negligible relaxation of the second neighboring $\mathrm{C}$ atoms. As the second neighboring carbon atoms around the vacancy have a closed electronic shell, the additional delocalized charge, which originates from first neighboring atoms, has no effect on the ion-ion screening of the second neighboring atoms.

\section{Conclusion}

Using ab-initio DFT calculations, we explained the lattice relaxation around diamond vacancies based on electron delocalization. Our proposed microscopic picture was based on the effect of the electron delocalization on the NN ion-ion screening and the bond length of back bonds. The calculated variation of charge (spin) localization on neighboring atoms with lattice relaxation contrasts with the inward relaxation of NN atoms around different charge states of the vacancy. Furthermore, the calculated magnitude of vacancy electron densities on neighboring atoms are in good agreement with available EPR measurements. Our calculated charge localizations on the first neighboring atoms of $V^{+}$disagree with tentative assignment of the NIRIM-3 EPR center to the positively charged vacancy in semiconducting diamond. The results suggest an outward lattice relaxation for symmetric displacement of $\mathrm{NN}$ atoms of vacancies in the semiconductor lattice.

\section{References}

1. G. Davies, in Properties, Growth and Application of Diamond, edited by M.H. Nazare, A.J. Neves (IEE, London, 2001), p. 193 
2. D.J. Twitchen, M.E. Newton, M. Baker, V.A. Nadolinny, in Properties, Growth and Application of Diamond, edited by M.H. Nazare, A.J. Neves (IEE, London 2001), p. 208

3. U. Gerstmann, M. Amkreutz, H. Overhof, Phys. Rev. B 60, R8446 (1999)

4. S.J. Breuer, P.R. Briddon, Phys. Rev. B 51, 6984 (1995)

5. A. Zywietz, J. Furthmüller, F. Bechstedt, Phys. Stat. Sol. (b) 210, 13 (1999)

6. U. Gerstmann, H. Overhof, Physica B 308, 561 (2001)

7. M. Heidari Saani, M.A. Vesaghi, K. Esfarjani, Eur. Phys. J. B 39, 441 (2004)

8. J.E. Lowther, in Properties, Growth and Application of Diamond, edited by M.H. Nazare, A.J. Neves (IEE, London 2001), p. 179

9. M. Heidari Saani, M.A. Vesaghi, K. Esfarjani et al., Phys. Rev. B 71, 035202 (2005)

10. J.A. Vanwyk, O.D. Tucker, M.E. Newton, J.M. Baker, G.S. Woods, P. Spear, Phys. Rev. B 52, 12657 (1995)

11. Gaussian 98, Revision A.7, M.J. Frisch et al., Gaussian, Inc., Pittsburgh PA, 1998
12. R. Jones, P.R. Briddon, in Identification of defects in seniconductors, edited by M. Stovela, Semiconductors and semimetals (Academic Press, Boston, 1998), Vol. 51A, Chap. 6

13. A.H. Edwards, W.B. Fowler, Phys. Rev. B 41, 10816 (1990)

14. J.P. Goss, Ph.D. thesis, University of Exter (1997)

15. A.D. Becke, J. Chem. Phys. 98, 5648 (1993)

16. J. Isoya, H. Kanda, Y. Uchida, S.C. Lawson, S. Yamasaki, H. Itoh, Y. Morita, Phys. Rev. B 45, 1436 (1992)

17. A.T. Collins, Diam. Relat. Mattr. 8, 1455 (1999)

18. J. Isoya, H. Kanda, Y. Morita, Proc. 4th Int. Conf. on new diamond science and technology, edited by S. Saito, N. Fujimori, O. Fukunaga, M. Kamo, K. Kobasho, M. Yoshikawa MYU, Tokyo, Japan (1994), p. 143

19. A. Mainwood, J. Phys. C. Solid State Phys. 12, 2443 (1979)

20. L.H. Li, J.E. Lowther, Phys. Rev. B 53, 11277 (1996) 